\title{
SA-I-02 Structures of Novel Nano-Materials \\ Determined by Synchrotron Radiation Powder Experiment
}

\author{
- Atoms in Fullerene Cages \& \\ Masaki Takata \& Makoto Sakata \\ JASRI/SPring-8, Nagoya University
}

Gas Molecules in Nano-Channel Microporous Compounds -

Recent development of nanotechnology is now stimulating the studies of creation of various novel materials with peculiar nano-scale structure by chemical and physical procedure. Among them, metallofullerene, which is a novel form of fullerene related material encapsulating metal atoms inside fullerene cage, is one of the most attractive nano-materials since we can invent new super molecule with novel specific electronic, chemical, physical properties by selecting the atoms encapsulated in fullerene cage.

Very recently, another interesting material, microporous coordination metal organic solids having uniform nano-sized channel structure, has successfully been created by self-assembly chemical reaction. The materials were found to show high performance gas adsorption and are expected as one of the next-generation gas storage materials. The extensive applications to physics, chemistry, medical science, environment engineering, energy industry, etc., are expected for these nano-materials in future.

For the production research and development of these nano-materials, the structure information is dispensable. However, it was very difficult to determine the position and structure of atoms and molecules trapped in the nano-sized space of fullerene cage and microporous by the conventional X-ray structure determination technique. Recently, a sophisticated analytical method, which is based on the information theory, the Maximum Entropy Method (MEM) using synchrotron radiation powder data[1], has been successfully applied to find metal atoms in fullerene cage[1,2] and gas molecules adsorbed in nano-channel microporous compounds[3]. The obtained structural information is not only atomic arrangement but also electron density distribution. The revealed MEM electron densities provide key information for better understanding of production mechanism and/or specific properties for these nano-materials. In the talk, the various peculiar structures of the novel nano-materials will be presented.

1) M. Takata, et al.: Nature 377 (1995) 46.

2) M. Takata, et al. : Phys.Rev.Lett. 78 (1997) 3330.; M. Takata, et al.: Phys.Rev.Lett. 83 (1999) 2214.; C.-R. Wang, et al.: Nature 408(2001)426; C.-R.Wang, et al., Angew. Chem. Int. Ed. 40(2001)397; E.Nishibori et al. , Angew. Chem. Int. Ed. 40(2001)2998

3) R.Kitaura et al.:Science 298(2002)2358.
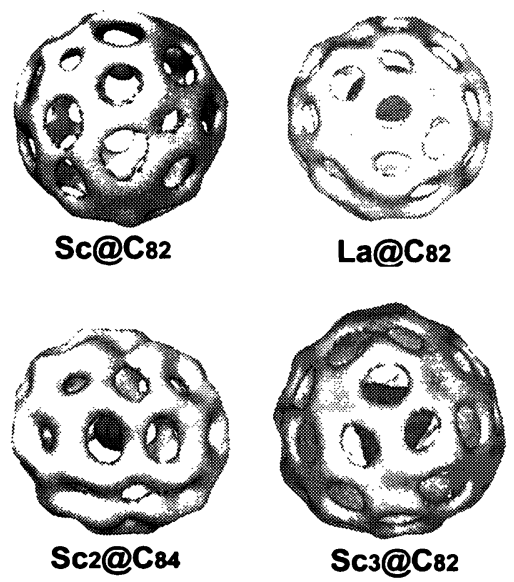

MEM Charge Desnties of Metallofullerenes

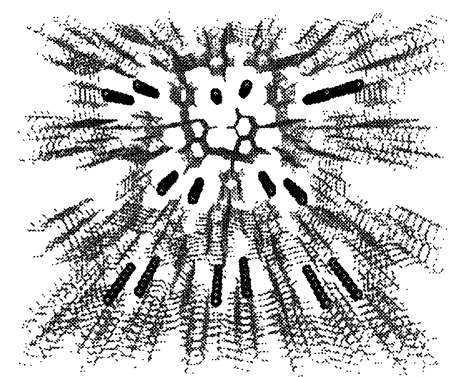

$\mathrm{O}_{2}$ Molecules Physisorbed in Nano-channel Microporous Compounds, $\left[\mathrm{Cu}_{2}(\mathrm{pzdc})_{2}(\mathrm{pyz})\right]_{\mathrm{n}}$ 